\title{
Lessons learned from wet washing the 6.5m MMT primary mirror in-situ
}

Ricardo Ortiz, Joseph T. Williams, William Goble

Ricardo Ortiz, Joseph T. Williams, William Goble, "Lessons learned from wet washing the 6.5m MMT primary mirror in-situ," Proc. SPIE 10700, Groundbased and Airborne Telescopes VII, 1070004 (6 July 2018); doi: $10.1117 / 12.2314348$

SPIE Event: SPIE Astronomical Telescopes + Instrumentation, 2018, Austin, Texas, United States 


\title{
Lessons Learned from Wet Washing the 6.5m MMT Primary Mirror In-situ \\ Ricardo Ortiz, Joseph T. Williams, William Goble \\ MMT Observatory, 1540 E. Second Street, University of Arizona, Tucson, AZ USA 85721-0064
}

\begin{abstract}
After gathering spectrophotometer data from the $6.5 \mathrm{~m}$ primary mirror at the MMT Observatory (MMT) for over ten years, the MMT has developed a soap and water wash procedure that effectively removes contaminates from the surface of the mirror without damaging the bare aluminum coating. While the in-situ soap and water wash requires a small amount of telescope downtime, these washes are still scheduled to take place every six months. The frequency of the washing was selected to keep the mirror performance as close to a fresh coating as possible throughout the year and to extend the recoating interval without allowing the reflectivity degrading more than $3 \%$ from a fresh coating. After being in service for almost two years, the spectrophotometer measurements indicate the 2016 primary mirror coating is on-track to maintain the specified reflectance for five years. This paper outlines the soap and water wash procedure developed for the MMT primary mirror and presents spectrophotometer data throughout the life of the 2005 and the 2016 mirror coatings.
\end{abstract}

Keywords: MMT Observatory, aluminum coatings, reflectance, scattering, $\mathrm{CO}_{2}$ cleaning, mirror washing

\section{INTRODUCTION}

This study summarizes the techniques, results, and lessons learned at the MMT to maintain a highly reflective aluminum coating on the $6.5 \mathrm{~m}$ primary mirror while maximizing the time between recoating. In conjunction with $\mathrm{CO}_{2}$ snow cleanings, the MMT has developed a process to wash the primary mirror with soap and water. Since recoating the primary mirror in 2016, the MMT has had the goal of soap and water washing the primary mirror every six months. The MMT is attempting to increase the recoating interval to five years as compared to the two-year recoating cycle followed by $\mathrm{LBTO}^{3}$ and Magellan ${ }^{4}$. While bare aluminum will naturally oxidize to create a surface layer of $\mathrm{Al}_{2} \mathrm{O}_{3}$, a significant amount of coating degradation is related to surface contaminates; the coating degradation is noticeable in the reflectance (R) and the diffuse scatter (S) measurements made by a Konica Minolta ${ }^{5}$ CM-600d spectrophotometer. Developed through many iterations, the present wash procedure is detailed below. Additionally, the spectrophotometer data illustrates the benefits of the soap and water washing, especially compared to just $\mathrm{CO}_{2}$ snow cleaning. By soap and water washing the primary mirror on a regular basis, the 2016 coating is almost as reflective as a fresh aluminum coating even after being in service for almost two years.

\section{BACKGROUND}

Due to site and budget constraints, the converted $\mathrm{MMT}^{[1]}$ was designed to perform primary mirror coatings while the mirror is still installed in the telescope (in-situ mirror coating), and this requires a large amount of effort and cost per coating to complete. For example, a typically coating will require over $\$ 60,000$ of expenses and ten weeks of telescope downtime. These expenses include crane and other equipment rental, transportation costs, and supplies (filaments, liquid nitrogen, cleaning chemicals, etc.). To support the coating process, ten full time employees are typically necessary for the entire shutdown period. The MMT primary mirror process is documented in "Re-aluminizing of the $6.5 \mathrm{~m}$ primary mirror at the MMT Observatory" ${ }^{\text {"2] }}$ and "In-situ Aluminization of the MMT 6.5m Primary Mirror" ${ }^{[3]}$.

\footnotetext{
${ }^{1}$ rortiz@mmto.org; phone 1520-879-4556

${ }^{2}$ The MMT Observatory is a joint facility of the Smithsonian Institution and the University of Arizona.

${ }^{3}$ LBTO, Large Binocular Telescope Observatory, Tucson, AZ, USA, www.lbto.org

${ }^{4}$ Magellan Telescopes, Las Campanas Observatory, La Serena, Chile, www.lco.cl

${ }^{5}$ Konica Minolta Sensing Americas, Inc., Ramsey, NJ, USA, https://sensing.konicaminolta.us
} 
The expense of recoating has been a driving factor in the extended recoating schedule that the MMT has adopted. However, the condition of the primary mirror is extremely important, and various wash methods have been explored in order to keep the mirror reflectivity as near a fresh coating as possible. The MMT has a goal to maintain the primary mirror reflectance (from $400 \mathrm{~nm}$ to $700 \mathrm{~nm}$ ) to within $3 \%$ of the fresh aluminum coating throughout the life of the coating. An additional but complementary goal is the keep the diffuse scatter of the primary mirror (as measured by the CM-600d spectrophotometer) below $1.5 \%$. Based on experience with previous mirror coatings and on typical resource loads, the MMT is following a twice-a-year schedule for soap and water washing the primary mirror.

\section{SOAP AND WATER MIRROR WASH}

The goal of any mirror cleaning procedure is to remove as much dust and particles as possible without creating scratches in the surface of the coating. In order to achieve this at the MMT, facility water treated onsite with a rented tri-bed water filter system is applied continuously for the duration of the wash. A very mild soap ( $3 \%$ mixture Liquinox by Alconox $\left.{ }^{6}\right)$ is applied to the mirror surface with a portable sprayer before the dabbing process is started. High-quality coarse yarn cotton mops are used to dab the mirror during the cleaning process; premium cotton roofing mops that have been soaked in Liquinox and rinsed repeatedly appear to work well with no damage to the aluminum coating.

The process requires a total of three days; this includes leaving the telescope inoperable for two nights. The first day is used to prepare the telescope. This includes removing the astronomical instrument and installing plastic barriers around the mirror to direct the waste wash water to containment vessel. The lower edge of the plastic around the perimeter of the mirror is clamped in place by an inflatable seal that holds the plastic against the edge of the glass. The upper edge of the perimeter plastic is taped to the upper edge of the trunnion beam. Additional plastic is tapped to the upper edge of the Cassegrain; this directs all of the process water past the mirror cell into the containment vessel. On the morning of the second day, a crew of six people begin the soap and water wash. The washing is typically completed around lunchtime, and the afternoon of the second day is used to remove the wash plastic from the mirror and to remove the washing equipment from the telescope building. The third day is used to reinstall the astronomical instrumentation.

After the containment vessel and drain hose have been installed, the mirror is thoroughly rinsed with the filtered facility water. The soap solution is applied to the mirror using a portable sprayer. Three mops that have been soaked in the soap solution are used to dab the mirror in purely vertical motion; the mops are more susceptible to scratching the coating with contaminates if the mop is dragged across the surface in a typical mopping motion. Figure 1 shows the mop head after contacting the glass in a vertical motion; the pattern left in the soap shows how the mop strands pull back together after the mop has been lifted in a vertical manner. Two mops are used around the perimeter while a specially rigged third mop is used to reach the center of the mirror, figure 2. This third mop is attached to a longer pole; to support the weight of the mop head, a rope is attached near the mop head and routed through a pulley mounted to the secondary mirror hub. By pulling on the rope and using one end of the pole as a pivot, the mop head can be raised and lowered in a nearly vertical motion. The mirror is kept wet with filtered water and the soap and water solution throughout the dabbing process. Once the entire mirror surface has been dabbed, the mirror is rinsed with filtered water until the soap residue is no longer evident on the surface. This rinse will general take between five and ten minutes. Starting directly opposite of the water hose, two air nozzles working away from each other begin to dry the mirror, figure 3 . The compressed air supplied to the nozzles is provided by two $25 \mathrm{hp}$ air compressors and is scrubbed using a series of oil coalescing filters and desiccant dryers. The drying air receives the same treatment as the compressed air supplied to the primary mirror cell. Since drying process takes a significant amount of time, the portions of the mirror that have not been dried are carefully kept wet with filtered water. Once the air nozzles have dried roughly half the mirror, someone enters the Cassegrain from below and begins drying the area near the Cassegrain hole with delicate task wipers; the air nozzles tend to blow the puddled water around the Cassegrain hole back on area that has already been dried. Figures 4 and 5 show the waste water containment vessel under the mirror cell with a short ladder in the vessel to allow someone access to the front surface through the Cassegrain hole. An additional person is supporting the person drying the mirror by holding the box of wipers and providing a fresh box of wipers as needed. After the mirror and the wash plastic has been dried, the staff generally waits roughly two hours before beginning to remove the wash plastic. This gives a little extra time to allow any water remaining on the wash plastic or in the joint between the glass and the wash plastic a chance to dry; in the process of removing the plastic, some small droplets of water almost inevitably get flicked on to the dry surface of the glass.

\footnotetext{
${ }^{6}$ Alconox Inc., White Plains, NY, USA, www.alconox.com
} 
Before arriving at the present procedure, some other process techniques tried for washing the primary mirror were: 1) the horizon pointing mirror was rinsed with a non-contact low pressure spray wash $\left.{ }^{[4,5]}, 2\right)$ the horizon pointing mirror was rinsed with a household pressure washer ${ }^{[6]}$, and 3) an articulated man-lift was used to provide access the center of the zenith pointing mirror. The non-contact spray washes did not appear to provide the improvement in mirror reflectivity that contact washed provided. Additionally, water control was an issue. The articulated man-lift basket was very time consuming to position around the telescope structure.

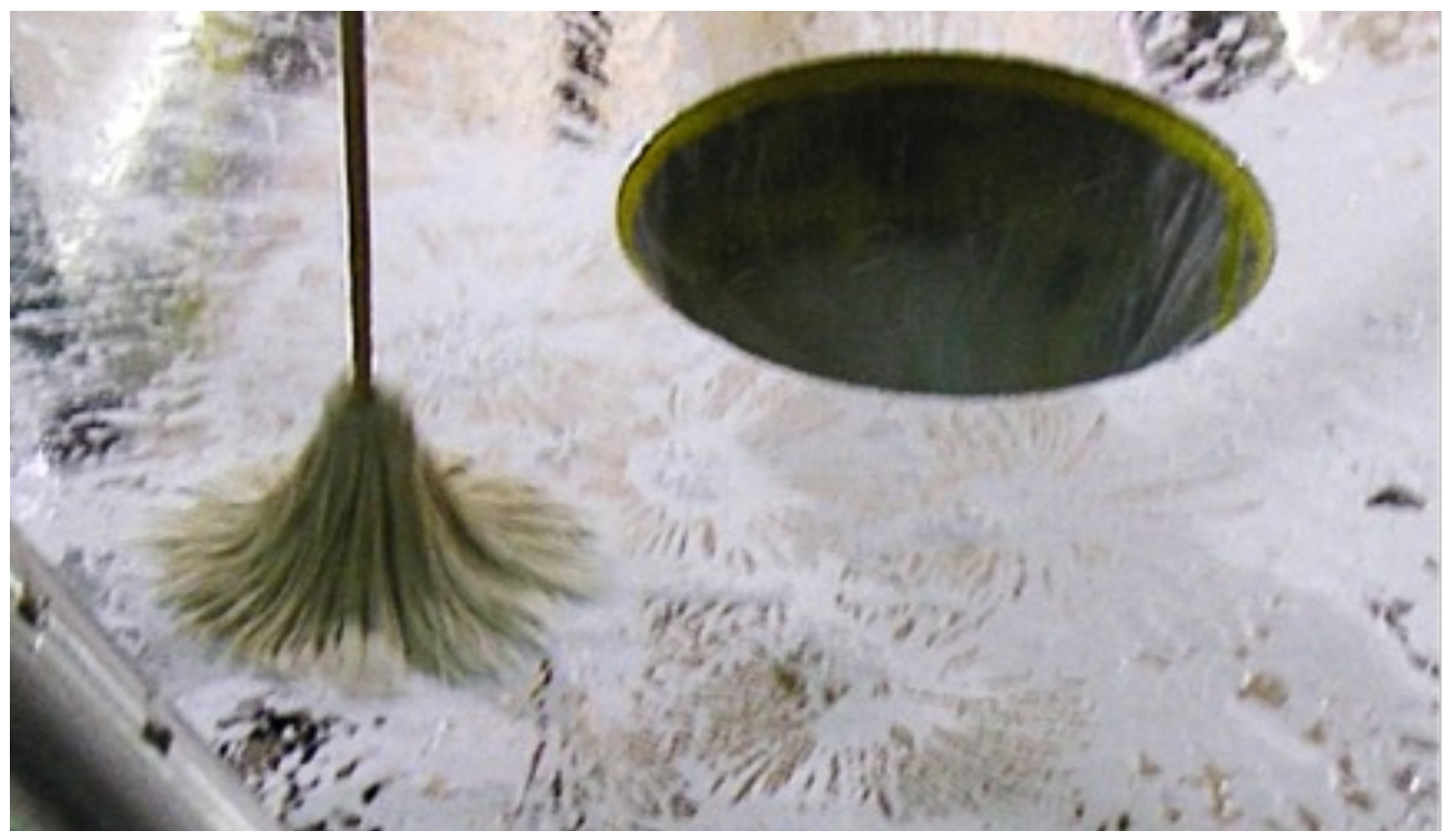

Figure 1. Image showing the up and down dabbing motion with a cotton mop. Photo is taken from the edge of the $6.5 \mathrm{~m}$ MMT primary mirror cell, looking towards the primary mirror surface and the Cassagrain. The mirror surface has been covered with a generous soap mixture.

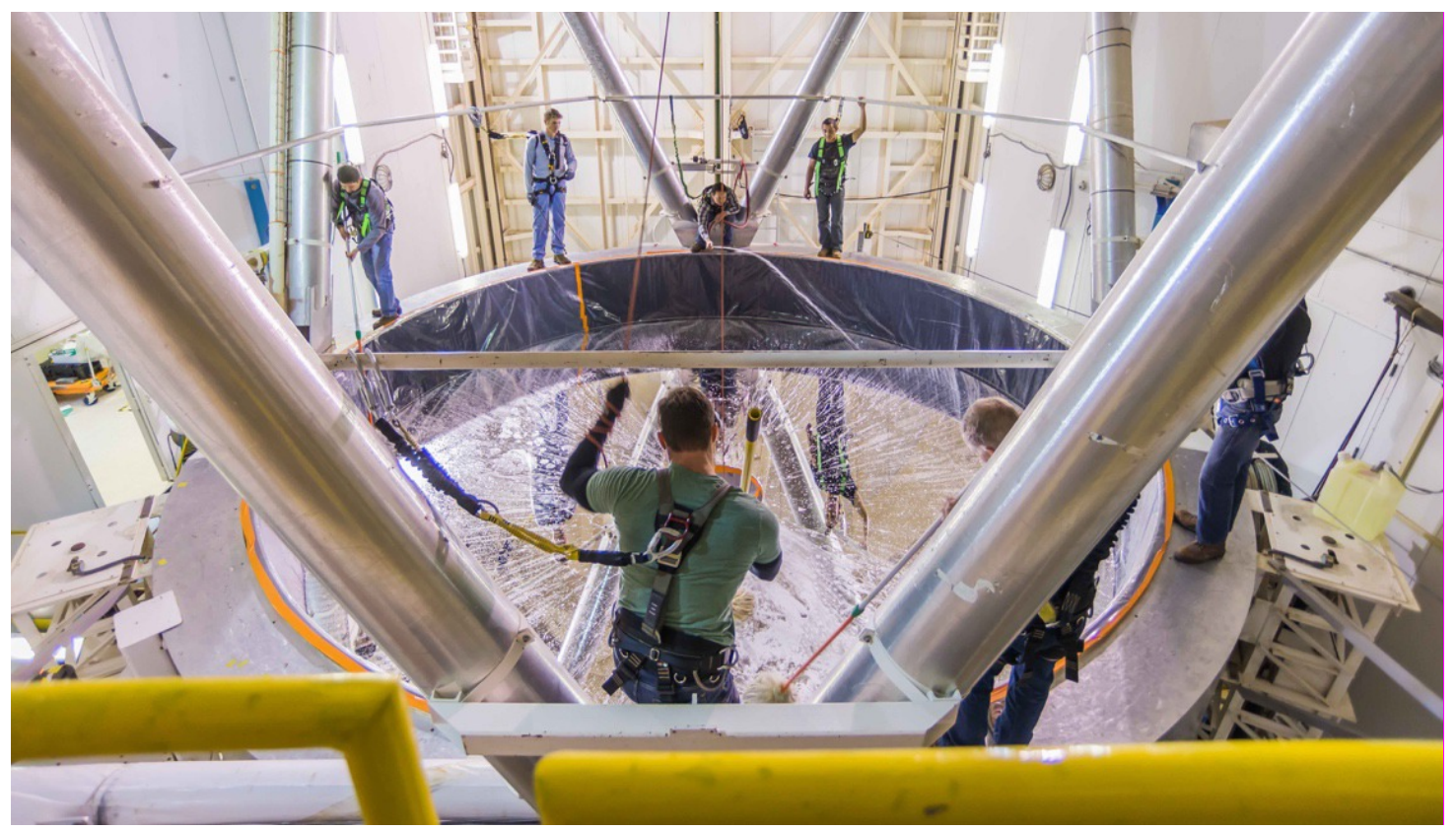

Figure 2. Dabbing process in progress, not visible is the pulley system attached to the secondary hub that allows one mop to reach the inner part of the mirror. Since personnel are working at a height of $5 \mathrm{~m}$, fall protection harnesses are required. 


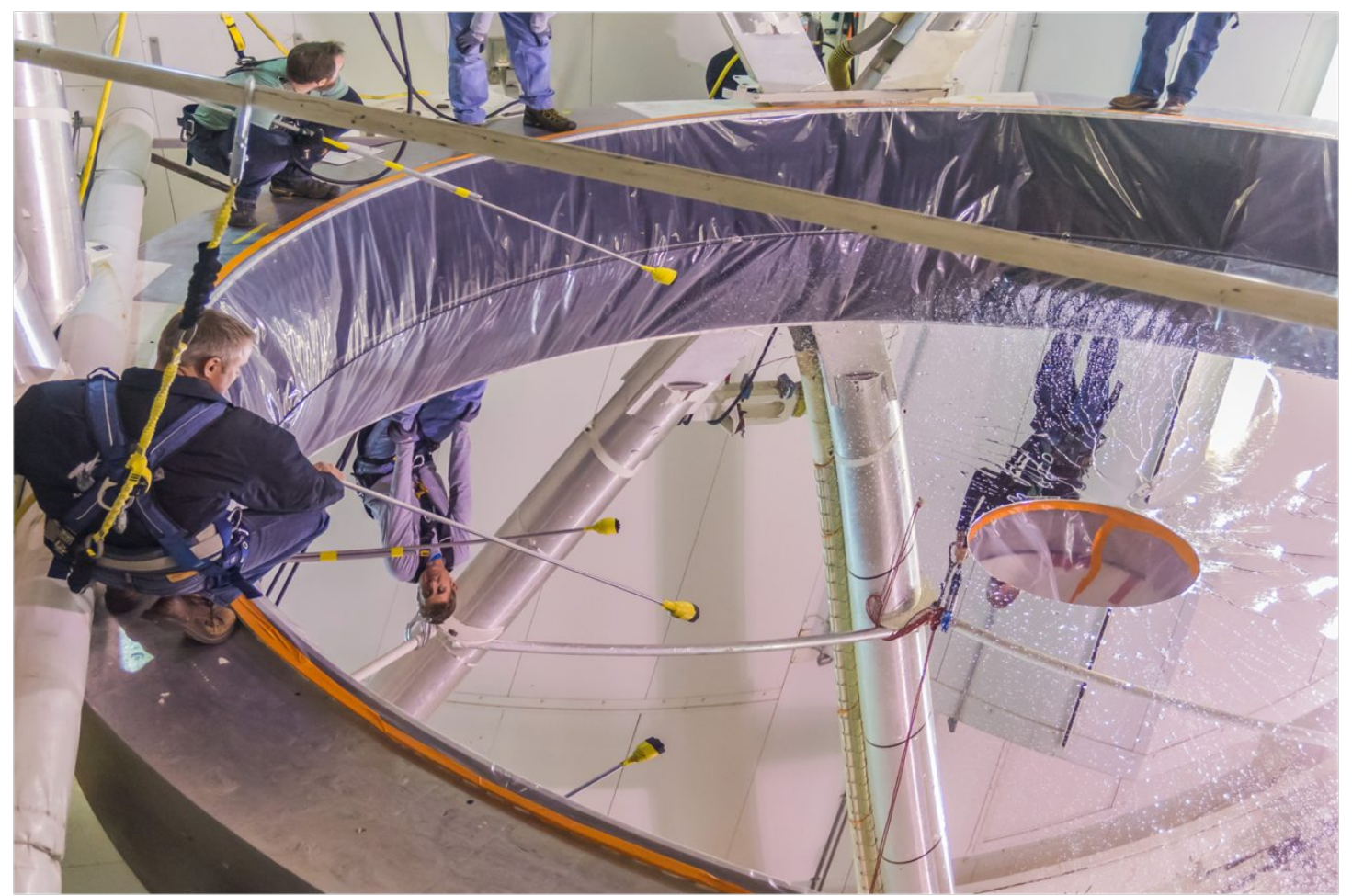

Figure 3. Drying the mirror surface. The air nozzles are used to working around the perimeter of the mirror towards the Cassegrain hole.
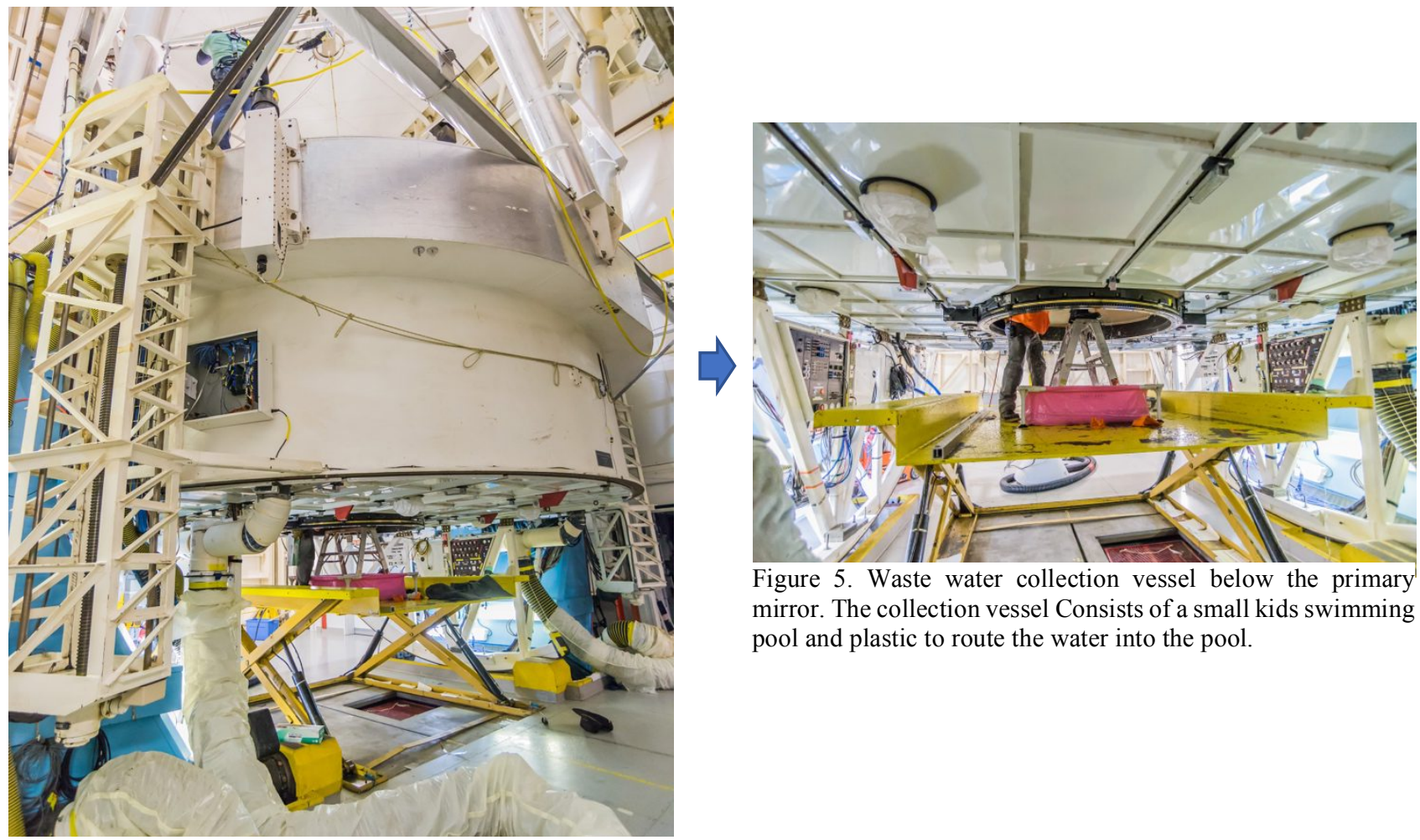

Figure 5. Waste water collection vessel below the primary mirror. The collection vessel Consists of a small kids swimming pool and plastic to route the water into the pool.

Figure 4. Overall view from chamber floor 


\section{SPECTROPHOTOMETER RESULTS}

\subsection{Data collection}

The Konica Minolta CM-600d Spectrophotometer measures and records the spectral properties of a sample every 10nm between the wavelength range of $400 \mathrm{~nm}$ to $700 \mathrm{~nm}$. The spectrophotometer automatically switches from a specular component included (SCI or reflectance) measurement mode and a specular component excluded (SCE or scatter) measurement mode. The CM-600d is designed to contact the surface being measured; this can cause minor but noticeable scratching of an aluminum coating. For the MMT primary mirror, measurements are taken at random in the lower section of the primary mirror as shown in figure 6 . This region was selected primarily for ease of access and to allow measurements to be taken while an astronomical instrument is installed. In order to reduce measurement variation, a Hemmendinger Color Laboratory ${ }^{7}$ (HCL) second surface aluminum coating standard is measured before and after a series of primary mirror spectrophotometer data is taken. The HCL standard measurements are compared to the manufacturer's specified values, and the mirror reflectance measurements are corrected accordingly. These are most often very small corrections. The instances the HCL reflectance measurements have been completely erroneous have often been due to the spectrophotometer not being in thermal equilibrium with telescope environment. For over ten years, spectrophotometer measurements of the primary mirror have been taken before and after any cleaning procedure has been performed, such as $\mathrm{CO}_{2}$ snow cleaning, water rinse, soap and water wash, and recoating. The measurement techniques and the cleaning processes were still in development during the life of the 2005 primary mirror coating, so only a limited amount of data from this coating is presented. Unfortunately, issues with the 2010 coating (briefly described in [2]) have made the spectrophotometer data from this coating not truly representative of a typical bare aluminum coating, and this data has not been presented. In 2016, a high quality aluminum coating was deposited on the MMT primary mirror, and the spectrophotometer data from this coating are the focus of the present analysis.

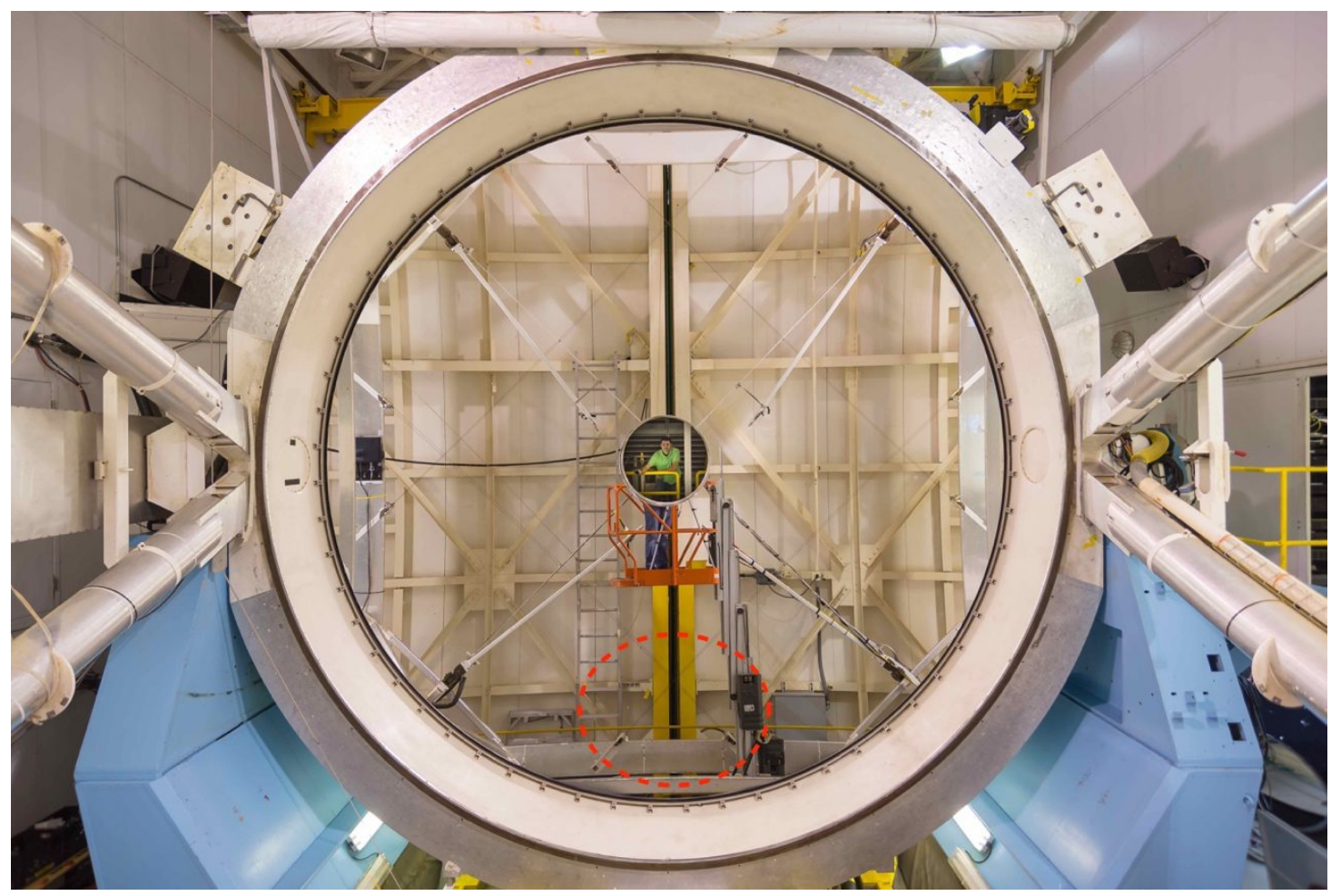

Figure 6. The 6.5m MMT horizon pointing after a fresh aluminum coating. Highlighted zone in the 6:00 area indicates location used at random for measurements using the Konica Minolta CM-600d Spectrophotometer

\footnotetext{
${ }^{7}$ Hemmendinger Color Laboratory, Tatamy, PA, USA
} 


\subsection{Results}

Measurements of the pristine 2016 coating were taken shortly after the coating chamber was opened; these reflectance and scatter values are baseline for the condition of the coating. The mirror had been washed approximately every six months after the coating, and the most recent wash of November 30, 2017 restored reflectance of the MMT primary mirror to within $0.8 \%$ of pristine values across the visible spectrum, figure 7 . As is typical, reflectance at the lower wavelengths $(400 \mathrm{~nm})$ degrade more than at the higher wavelengths. Not only are surface contaminates being, but the wash process itself does not appear to be degrading the mirror coating. The scatter measurements after the November 2017 was also illustrate the improvement in the coating performance from the soap and water wash, figure 8.

In the absence of a significant event ${ }^{8}$ that damages the primary mirror coating, the reflectance results from the first three soap and water washings (figure 9) indicate the coating reflectance is on track to remain below the $3 \%$ degradation threshold for an estimated five-year coating life. The soap and water washing will continue to be scheduled for six-month intervals However, the actual duration between soap and water washing may vary by a few months depending on other scheduling constraints.

As illustrated in figure 10, a CO 2 snow cleaning is less effective than a soap and water wash. However, $\mathrm{CO} 2$ cleaning does not require any telescope downtime and can be completed on much more frequent basis, roughly every two weeks. Hence, $\mathrm{CO} 2$ cleaning of the primary mirror will continue to take place throughout the life of the coating.

A comparison of the 2005 and 2016 coatings are included (see Table 1 and 2). Data from the 2005 coating illustrate a soap and water wash can produce a significant improvement in the coating performance at any point in the life of a coating. However, the data illustrates the 2005 coating would have exceeded the 3\% degradation threshold after the May 2008 wash. This may describe the importance of maintaining a rigorous wash schedule throughout the life of the coating, for frequent soap and water washes were not started until over two years after the 2005 coating had been deposited.

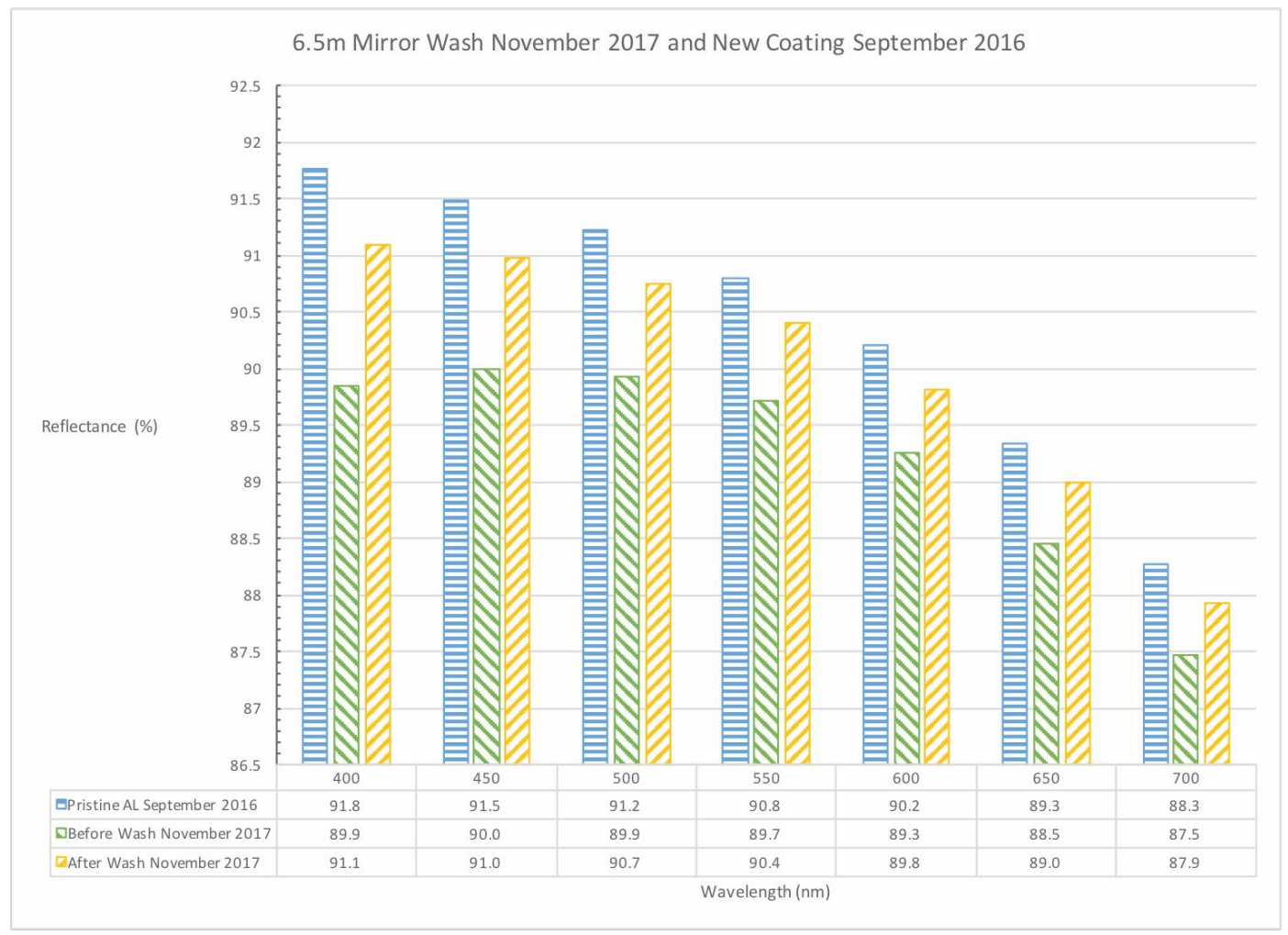

Figure 7. Reflectance $(\boldsymbol{R})$ of aluminum coating deposited in September 2016 and washed during November 2017.

\footnotetext{
${ }^{8}$ A significant event could be: cooling the mirror below the dew point and allowing condensation to form on the coating, allowing a significant amount of water or other fluid to drip on the coating, a ringtail cat family living on the coating, etc.
} 


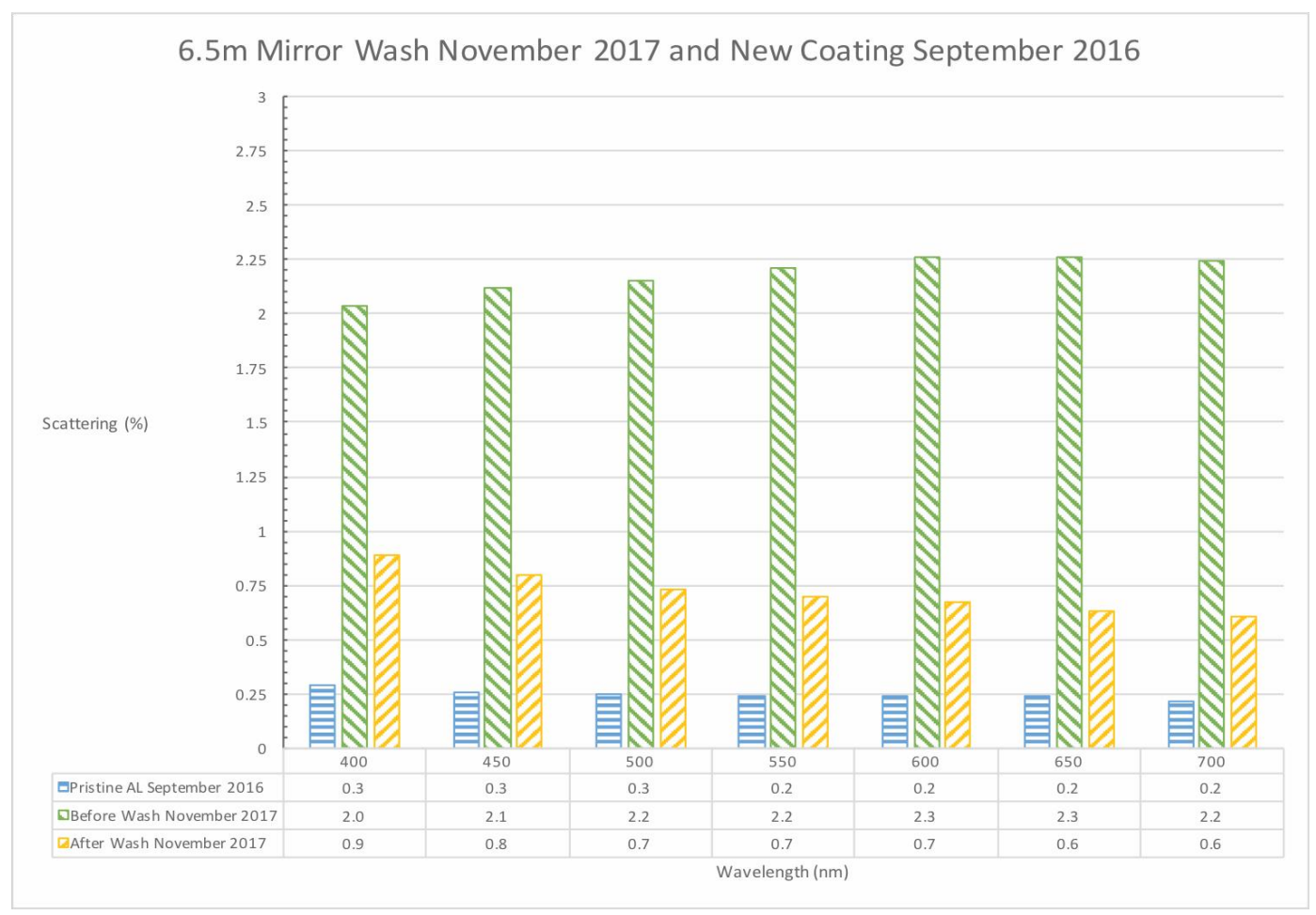

Figure 8. Scattering $(\boldsymbol{S})$ results of aluminum coating, evaporated on the mirror's surface during September 2016 and washed during November 2017. The chart shows $\boldsymbol{S}$ of the primary mirror after performing the third wash of the 14-month old coating.

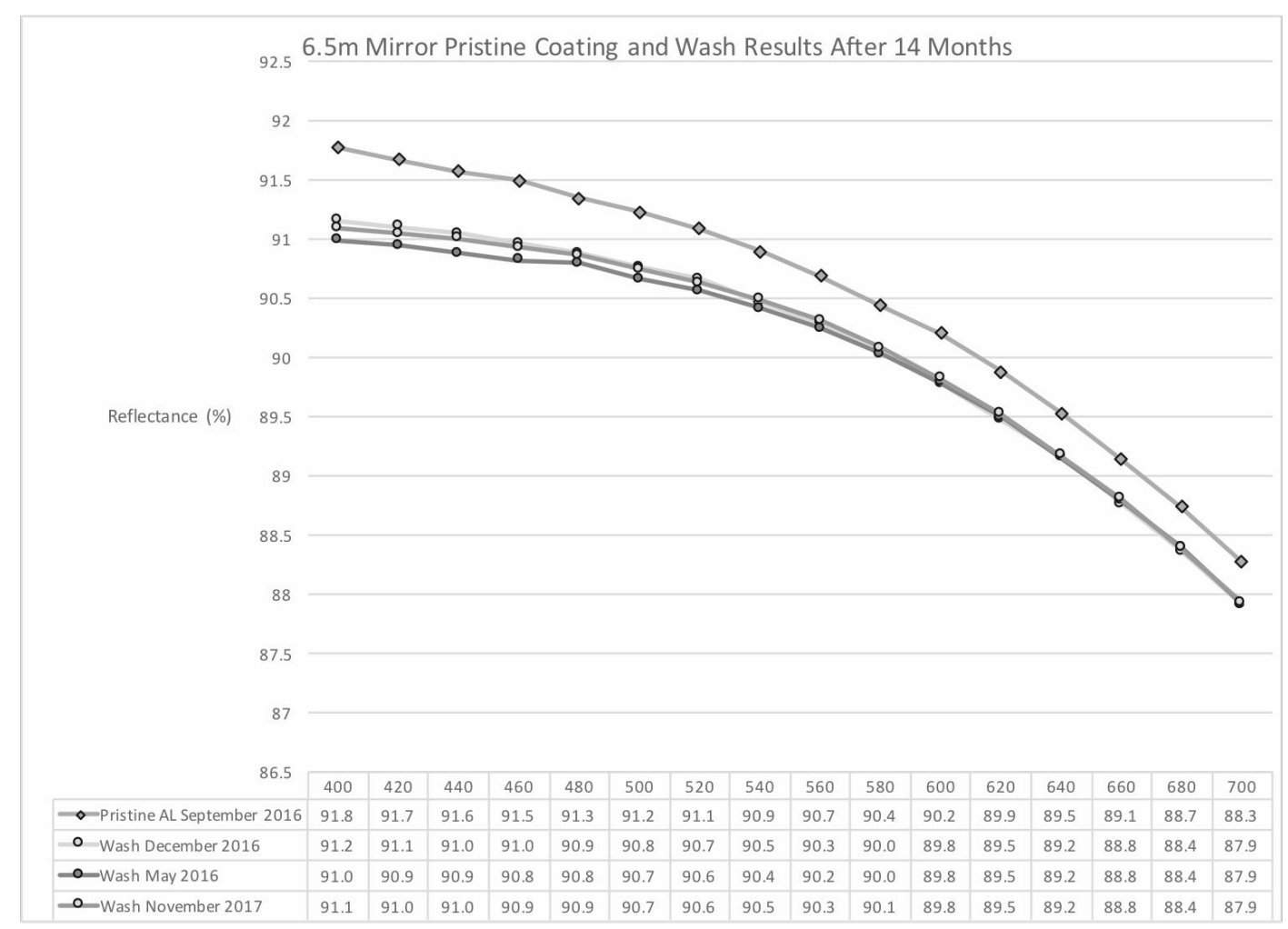

Figure 9. Results for the latest coating ascertains negligible damage occurs to the coating from the dabbing process. 


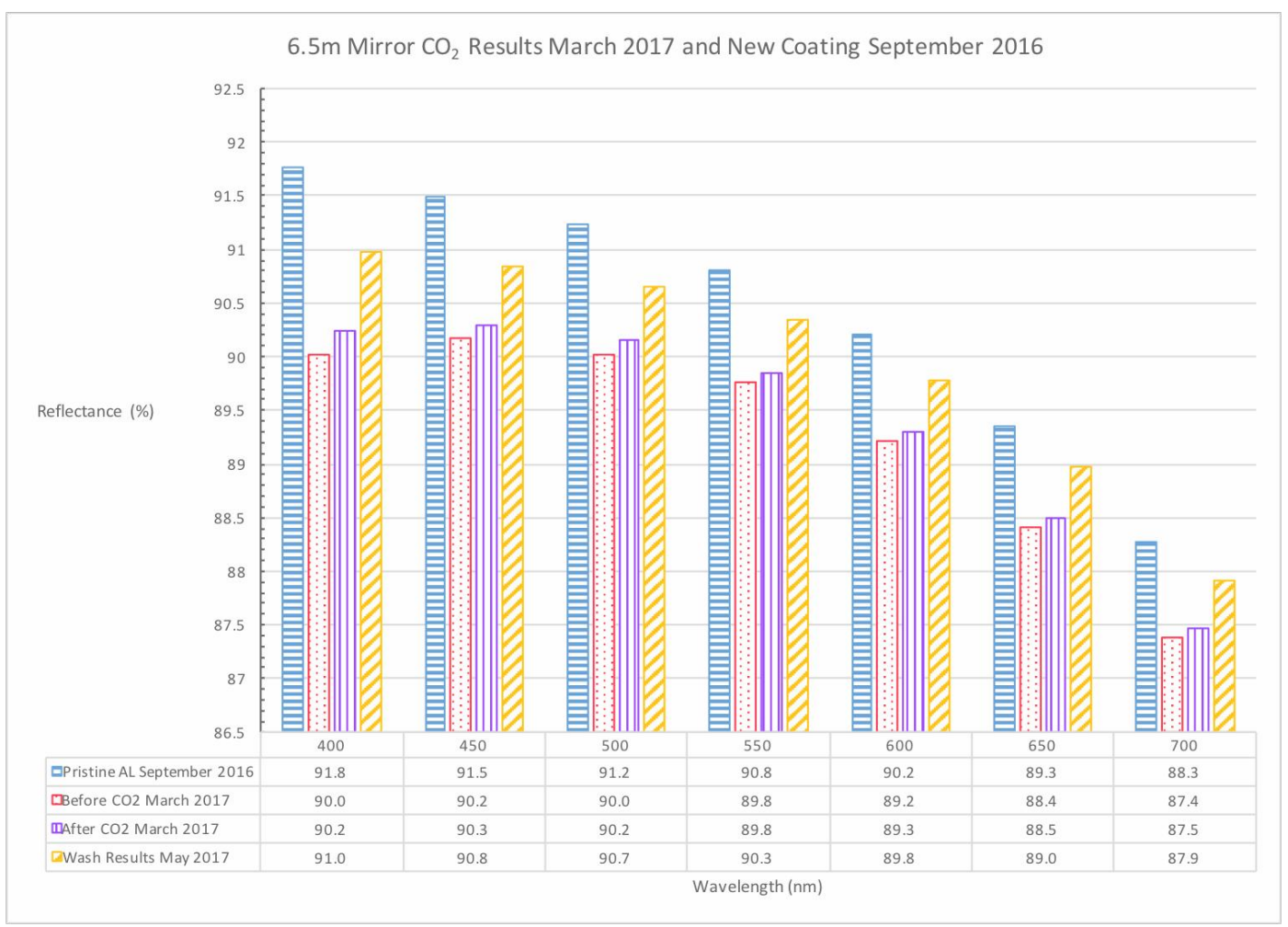

Figure 10. The reduced effectiveness of $\mathrm{CO}_{2}$ cleaning compared to cotton dabbing wash is evident.

Table 1. Reflectance $(R)$ wash results for the 2005 and 2016 coatings at the blue spectrum (400nm) of the Minolta. Mirror wash was not fully established until 2007. Resulting in a 27 -month deterioration to the 2005 coating before the first wash. Notice how after 43 -month before wash $R$ had degraded down to $85 \%$ and but recovered significantly.

\begin{tabular}{|c|c|c|c|c|c|c|c|c|}
\hline \multirow{3}{*}{ Reflectance $(R)$} & \multicolumn{4}{|l|}{$\begin{array}{l}\text { New } \\
\text { Coating } \\
2005 \\
\end{array}$} & \multicolumn{4}{|l|}{$\begin{array}{l}\text { New } \\
\text { Coating } \\
2016 \\
\end{array}$} \\
\hline & September & December & May & April & September & December & May & November \\
\hline & - & 2007 & 2008 & 2009 & - & 2016 & 2017 & 2017 \\
\hline Months between wash & - & 27 & 5 & 11 & - & 3 & 5 & 6 \\
\hline Coating age (months) & 0 & 27 & 32 & 43 & 0 & 3 & 8 & 14 \\
\hline Initial $R @$ 400nm & 91.7 & 87.5 & 88.0 & 84.8 & 91.8 & 90.7 & 90.4 & 89.9 \\
\hline Afterwash $R @ 400 \mathrm{~nm}$ & - & 89.2 & 88.5 & 88.4 & - & 91.2 & 91.0 & 91.1 \\
\hline Improvement in $R$ & - & 1.7 & 0.5 & 3.6 & - & 0.5 & 0.6 & 1.2 \\
\hline
\end{tabular}


Table 2. Scattering $(S)$ wash results for the 2005 and 2016 coatings at the blue spectrum $(400 \mathrm{~nm})$ of the Minolta. Observe how starting early in the life of a pristine coating maintains a low $S$ value. Data from 2005 shows a contaminated coating will not recover below $1 \%$

\begin{tabular}{|c|c|c|c|c|c|c|c|c|}
\hline \multirow{3}{*}{ Scattering $(S)$} & \multicolumn{4}{|l|}{$\begin{array}{l}\text { New } \\
\text { Coating } \\
2005 \\
\end{array}$} & \multicolumn{4}{|l|}{$\begin{array}{l}\text { New } \\
\text { Coating } \\
2016 \\
\end{array}$} \\
\hline & September & December & May & April & September & December & May & November \\
\hline & - & 2007 & 2008 & 2009 & - & 2016 & 2017 & 2017 \\
\hline Months between wash & - & 27 & 5 & 11 & - & 3 & 5 & 6 \\
\hline Coating age (months) & 0 & 27 & 32 & 43 & 0 & 3 & 8 & 14 \\
\hline Initial $S @ 400 \mathrm{~nm}$ & 0.3 & 7.7 & 2.6 & 4.2 & 0.3 & 1.0 & 1.2 & 2.0 \\
\hline After wash $S @ 400 \mathrm{~nm}$ & - & 1.4 & 1.4 & 1.5 & - & 0.4 & 0.7 & 0.9 \\
\hline Removal of $S$ & - & -6.3 & -1.2 & -2.8 & - & -0.6 & -0.5 & -1.1 \\
\hline
\end{tabular}

\section{CONCLUSIONS}

The MMT has developed an effective in-situ soap and water for the $6.5 \mathrm{~m}$ primary mirror. On surface that is generously wet with the soap solution, the dabbing process using high quality cotton mops allows access to the entire mirror surface, and the dabbing process has not damaged the bare aluminum coating in any manner. The spectrophotometer data collected since 2004 has demonstrates the effectiveness of a soap and water washing especially compared to $\mathrm{CO}_{2}$ snow cleaning. Although two nights of telescope downtime are required, the soap and water washes are being scheduled for every six months. This wash interval was selected to keep the reflectance of the coating as close to pristine as possible throughout the year and to hopefully extend the life of the primary mirror coating to five years while keeping the reflectivity within $3 \%$ of a fresh coating.

\section{REFERENCES}

[1] West, S. C., S. Callahan, F. H. Chaffee, W. Davison, S. DeRigne, D. Fabricant, C. B. Foltz, J. M. Hill, R. H. Nagel, A. Poyner, J. T. Williams, "Toward first light for the 6.5-m MMT telescope,", MMT Observatory Technical Report 30 (1997).

[2] W. Goble, "Re-aluminizing of the 6.5m primary mirror at the MMT Observatory," MMT Observatory, SPIE 10700155 (2018)

[3] W. Kindred, "In-situ Aluminization of the MMT 6.5m Primary Mirror", MMT Observatory, SPIE 6273-109 (2006)

[4] W. Kindred, "Effects of $\mathrm{CO}_{2}$ Cleaning and Detergent Washing on the Specular and Diffuse Reflectance of the MMT 6.5m Primary Mirror," MMT Observatory Internal Technical Memorandum 02-1 (2002).

[5] W. Kindred, "Effects of $\mathrm{CO}_{2}$ Cleaning and Detergent Washing on the Specular and Diffuse Reflectance of the MMT 6.5m Primary Mirror, An Addendum to MMTO ITM 02-1," MMT Observatory Internal Technical Memorandum 03 $6(2003)$.

[6] W. Kindred, "MMT Primary Mirror Wash Results of May 11,2004," MMT Observatory Internal Technical Memorandum 04-2 (2004). 